\title{
Integrating Optical Cavities in Ion Traps for Scalable Quantum Information Networks
}

\author{
Nina Podoliak ${ }^{1}$, Hiroki Takahashi ${ }^{2}$, Matthias Keller ${ }^{2}$, Peter Horak ${ }^{1 *}$ \\ ${ }^{1}$ Optoelectronics Research Centre, University of Southampton, Southampton SO17 1BJ, United Kingdom \\ ${ }^{2}$ Department of Physics and Astronomy, University of Sussex, Falmer, BN1 9QH, United Kingdom \\ peh@orc.soton.ac.uk
}

\begin{abstract}
We investigate the distortions of trapping potentials when integrating fiber-tip optical cavities into small ion traps. Trap depths, frequencies, and anharmonicity are investigated numerically for several geometries and the most stable traps are identified.

OCIS codes: (270.5585) Quantum information and processing; (060.5565) Quantum communications; (350.4600) Optical engineering.
\end{abstract}

\section{Introduction}

Radiofrequency (rf) traps for single or few atomic ions are currently one of the leading contenders for quantum information processing (QIP), mainly due to their unsurpassed long decoherence time [1]. Scaling QIP to many quantum bits will however ultimately require connecting individual ion traps either by shuffling ions between traps [2] or, alternatively, by optical fiber links [3]. For the latter approach, coupling ions to optical fiber-coupled cavities provides, at least in principle, a deterministic ion-photon interface that would allow for much faster photon-mediated ion-ion QIP than current probabilistic schemes [4], provided that the strong cavity coupling regime can be achieved. While this has been demonstrated for single neutral atoms and collectively for many atoms, strong coupling of single ions to optical cavities is still to be achieved.

The main design challenge for such ion traps with integrated optical resonators lies in the fact that small optical cavity volumes are required to achieve strong coupling and thus the dielectric mirrors forming the cavity have to be placed close to the ion trap center. These dielectrics, however, then distort the electromagnetic (rf) fields that form the ion trap and thus may significantly degrade their performance [5]. Here we investigate a range of ion traps and optical cavity configurations to identify the most suitable geometries to achieve stable rf ion trapping together with strong ion-cavity coupling.

\section{Methods}

For our numerical investigation we select five different ion trap geometries, two linear traps with blade-shaped and wafer electrodes, respectively, a cylindrical endcap trap, a cylindrical stylus trap, and a planar surface trap, as shown in Fig. 1. All traps are scaled to have typical electrode gaps of $1 \mathrm{~mm}$. The optical cavities are assumed to be formed between the tips of two dielectric cylinders $0.7 \mathrm{~mm}$ in diameter representing tips of optical fibers coated with dielectric mirrors [6], and cavity alignment along all three spatial axes of the ion traps is considered. For each of these ion-trap and cavity configurations we simulate the rf trapping fields $\mathbf{E}(\mathbf{r})$ using a full 3-dimensional finite element method (Comsol Multiphysics $\left.{ }^{\circledR}\right)$ and calculate the effective ion trapping potential

$$
\Phi(\mathbf{r})=\frac{q}{4 m \omega^{2}} \mathbf{E}(\mathbf{r})^{2}
$$

where $q$ is the ion charge, $m$ the ion mass, and $\omega$ the $\mathrm{rf}$ angular frequency. In all cases, the applied rf voltage is 200 $\mathrm{V}$ and the frequency is $12.7 \mathrm{MHz}$.

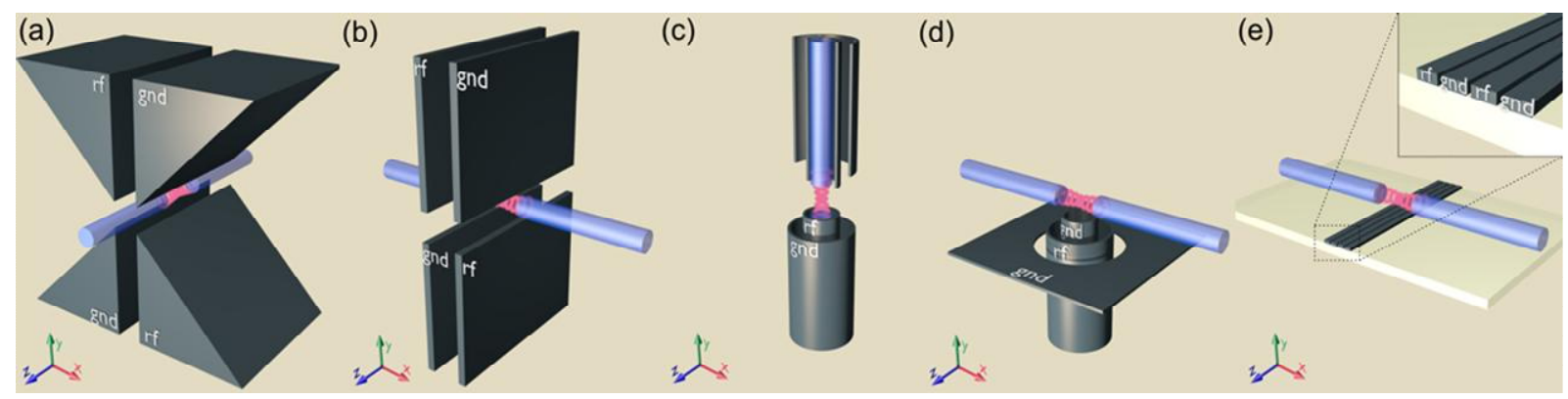

Fig. 1. Schematics of investigated ion traps: (a) blade, (b) wafer, (c) endcap, (d) stylus, and (e) surface trap. 


\section{Results and Discussion}
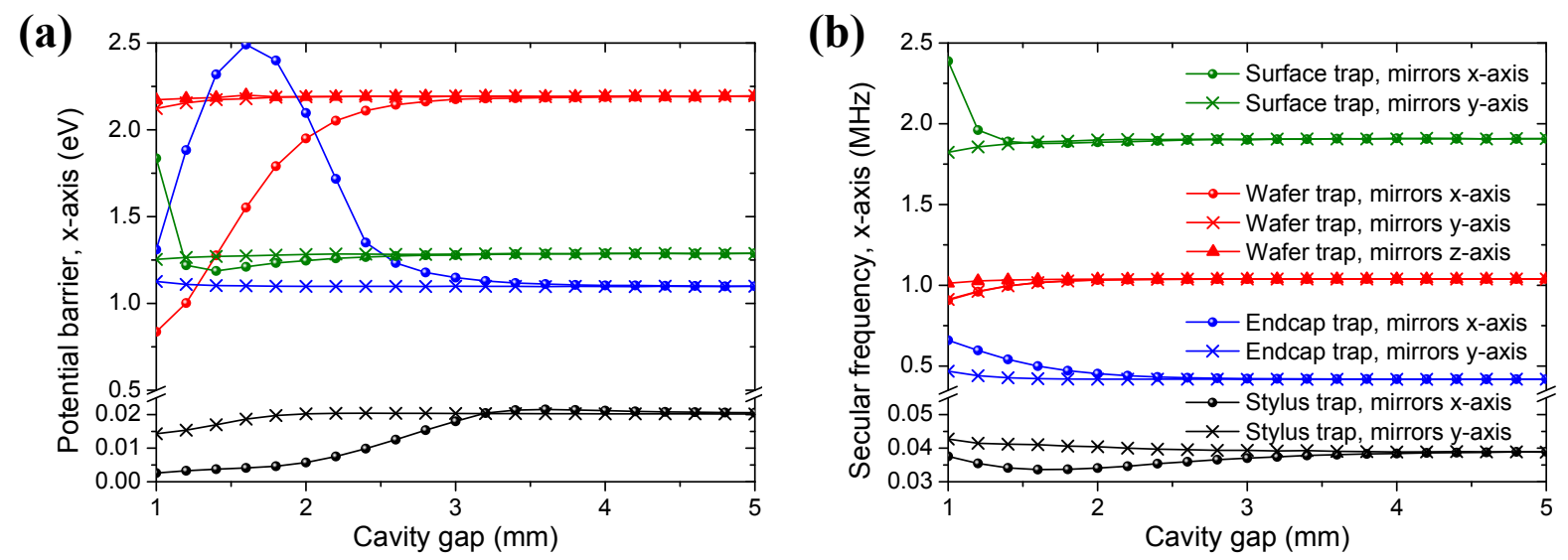

Fig. 2. Comparison between (a) potential barrier heights and (b) secular frequencies for different ion traps and different optical cavity orientations depending on the gap between cavity mirrors.

We characterize the effect of dielectric mirrors on various traps with various cavity mirror alignments by analyzing the trapping potential depths and secular frequencies along the trap-confining axes (x- and y- axis). Fig. 2 shows some sample results of the potential barrier heights and the trap frequency in the x-direction as a function of the separation between the mirrors. The blade trap (not shown in Fig. 2) behaves similarly to the wafer trap. We see a notable difference in trap depths and frequencies between the different geometries, with the linear traps generally providing better confinement than the cylindrical traps. Several trap/cavity geometries suffer major distortions when the dielectric mirrors are brought close to the trap center, e.g., we find a $60 \%$ reduction of trap depth along the $\mathrm{x}$ and $y$ - axis for the wafer trap with a cavity aligned along the corresponding axis, and a nearly complete loss of the trapping potential in the stylus trap with a cavity aligned along the x-axis. On the other hand, we find that trap distortions are minimized if the dielectric cavity mirrors are aligned along the main trap axis and either shielded by the electrodes (e.g., in endcap traps with cavities inside the electrodes along the y-direction) or placed along nodes of zero electric fields (e.g., in the linear traps with mirrors along the z-axis).

In further simulations we have also investigated the effects of misaligned geometries (i.e., where the cavity mirrors are not carefully aligned with the trap symmetry axes), of mirror materials (i.e., materials of low and high dielectric permittivity), and of surface charges on the mirrors as have been observed experimentally.

\section{Conclusion}

Our results show clearly how small dielectric optical cavities integrated into a range of ion traps can severely interfere with the trapping properties. The smallest distortions are found if the cavities are aligned along the major symmetry axis of the electrode geometries through either shielding or minimizing the electromagnetic field at the position of the dielectric mirrors. These configurations are therefore best suited for achieving small cavity volumes and thus large ion-photon coupling as required for scalable quantum information networks.

This work was supported by the UK Quantum Technology Hub in Networked Quantum Information Technologies (NQIT), EPSRC project no. EP/M013243/1. The data used here is available under the Creative Commons Attribution 4.0 License [7].

\section{References}

[1] T. P. Harty, D. T. C. Allcock, C. J. Ballance, L. Guidoni, H. A. Janacek, N. M. Linke, D. N. Stacey, and D. M. Lucas, "High-fidelity preparation, gates, memory, and readout of a trapped-ion quantum bit," Phys. Rev. Lett. 113, 220501 (2014).

[2] D. Kielpinski, C. Monroe, and D. J. Wineland, “Architecture for a large-scale ion-trap quantum computer,” Nature 417, 709-711 (2002).

[3] H. J. Kimble, "The quantum internet," Nature 453, 1023-1030 (2008).

[4] D. L. Moehring, P. Maunz, S. Olmschenk, K. C. Younge, D. N. Matsukevich, L.-M. Duan, and C. Monroe, "Entanglement of single-atom quantum bits at a distance," Nature 449, 68-71 (2007).

[5] M. Harlander, M. Brownnutt, W. Hänsel, and R. Blatt, “Trapped-ion probing of light-induced charging effects on dielectrics,” New J. Phys. 12, 093035 (2010).

[6] D. Hunger, T. Steinmetz, Y. Colombe, C. Deutsch, T. W. Hänsch, and J. Reichel, “A fiber Fabry-Perot cavity with high finesse,” New J. Phys. 12, 065038 (2010).

[7] University of Southampton eprints, http://eprints.soton.ac.uk/id/eprint/386420; doi: 10.5258/SOTON/386420 